Original

\title{
Psychometric properties and performance of the Oral Health Impact Profile (OHIP-14s-ar) among Sudanese adults
}

\author{
Nadia Khalifa1), Patrick F. Allen'2), Neamat H. Abu-bakr ${ }^{3)}$, \\ and Manar E. Abdel-Rahman ${ }^{4}$ \\ 1)Prosthodontic Department, Faculty of Dentistry, Khartoum University, Khartoum, Sudan \\ 2)Department of Restorative Dentistry, Cork University Dental School \& Hospital, Wilton, Ireland \\ 3)Conservation Department, Faculty of Dentistry, Khartoum University, Khartoum, Sudan \\ 4)Department of Statistics, Faculty of Mathematics, Khartoum University, Khartoum, Sudan
}

(Received November 5, 2012; Accepted March 16, 2013)

\begin{abstract}
The aims of this study were to develop a Sudanese-Arabic version of the English-language Oral Health Impact Profile-14 (OHIP-14), assess the psychometric properties and performance of this new instrument, and then use it to investigate the impacts of selected oral disorders on oral health-related quality of life (OHRQoL). Sudanese adolescents and adults (age, $\geq 16$ years; 781 males and 1107 females) attending outpatient clinics in Khartoum State were enrolled. The OHIP-14 was adapted, and the validity and reliability of the Sudanese-Arabic version of the questionnaire (OHIP-14s-ar) were assessed. The OHIP-14s-ar was then used to measure OHRQoL. Pearson correlation coefficients and multivariate analysis were used to examine relationships between OHRQoL and characteristics ascertained by interviews and clinical examinations. The OHIP-14s-ar had suitable construct validity, reliability, and internal consistency (i.e., Cronbach $\alpha$ ). More than half of the participants reported that oral disorders affected quality of life; psychological discomfort and physical pain were the most frequently reported oral health impacts. Our results suggest that being female, tooth decay, systemic illness, and dry mouth worsen OHRQoL. The oral disorder with the greatest impact was dental caries, and tooth condition seemed to be more important than absence of teeth. The OHIP-
\end{abstract}

Correspondence to Dr. Nadia Khalifa, Prosthodontic Department, Faculty of Dentistry, Khartoum University, P.O. Box 102, Khartoum, Sudan

Fax:+249-187557654 E-mail: nadiakhalifa@uofk.edu 14s-ar yielded convincing psychometric data, and its performance makes it a suitable measure for future cross-sectional and longitudinal studies in Sudan. (J Oral Sci 55, 123-132, 2013)

Keywords: oral health; quality of life; self-assessment; Sudanese adults.

\section{Introduction}

Clinical indicators are indispensable in assessing oral health. However, comprehensive assessments of oral health should include patient self-reports on oral health. Such assessment is necessary, as patient perceptions are likely to differ from those of health care professionals (1). Inclusion of subjective, as well as objective, health assessments would help in developing better strategies for health promotion, disease prevention programs, and distribution of health resources (2). As part of this effort, several oral health-related quality of life (OHRQoL) measures have been developed (3-9). While some measures of oral health status were developed to provide an index of the impact of oral disorders, others place functional disorders and their social consequences in a hierarchy of outcomes.

Slade and Spencer (10) developed and validated the original Oral Health Impact Profile (OHIP-49), which was based on Locker's conceptual model of oral health (11). This model linked oral disorders with biological, behavioral, and psychosocial consequences, using a framework from the World Health Organization (WHO) document, The WHO International Classification of 
Impairments, Disabilities and Handicaps (ICIDH) (12). The Oral Health Impact Profile questionnaire has welldocumented psychometric properties and has been used in both cross-sectional and longitudinal studies (13-15). It consists of 49 questions and measures seven dimensions, including functional limitations, physical pain, psychological discomfort, physical disability, psychological disability, social disability, and other handicaps. A shortened version of this profile, the (OHIP-14), consists of a 14-item questionnaire that has been developed and validated (16). Although the authors suggested that shortening the scale meant sacrificing some of the comprehensiveness of the original OHIP scale, its practical utility was improved. Its use in cross-sectional surveys has been documented, and its discriminant validity is well established $(13,17)$.

To date, there is little information on how oral disorders affect OHRQoL in African populations. To adequately address the oral health needs and concerns of Sudanese patients, it is essential to understand the impact of oral disorders on daily living and QoL by developing, or culturally adapting, a psychometric instrument such as the OHIP-14. We hope that this effort will increase understanding of how Sudanese patients perceive their oral health care needs and assist in identifying the oral health outcomes they seek.

We used a Sudanese-Arabic version of the Oral Health Impact Profile 14 (OHIP-14s-ar) to assess the impact of oral disorders on OHRQoL and identify factors associated with poorer OHRQoL.

\section{Materials and Methods}

\section{Study design}

This cross-sectional oral health survey was part of a study conducted from August 2009 through March 2010 that was designed to assess the functional and psychosocial impact of dental disease. Participants were recruited from individuals attending outpatient dental hospitals $(n$ $=1,659)$ and dental health centers $(n=229)$ distributed among the seven provinces (Um Durman, Khartoum Bahri, Khartoum, Jabal Aulia, Sharg En Nile, Karary, and Um badda) of Khartoum State. Sudan was the largest country in Africa before South Sudan became an independent country on 9 July 2011. It was divided into 25 states, and Khartoum State, the capital, was the most densely populated state. The study sample comprised 1,888 patients, divided into seven age groups: 16-24 ( $n$ $=431), 25-34(n=616), 35-44(n=368), 45-54(n=$ $253), 55-64(n=113), 65-74(n=77)$, and $75+$ years $(n$ $=22$ ). Sample size was calculated using the formula for proportion estimates, assuming a tooth loss prevalence of
$67 \%$ (as indicated by previous Sudanese studies) $(18,19)$ with a precision of 3 ; the design effect was set at 2 . The sampling frame for the study was the public dental service in Sudan. Health care systems in Khartoum have three levels (federal, state, and locality). All outpatient dental clinics in these facilities (which mainly provide basic dental services) were included in the sampling frame. The sample size of each outpatient clinic was obtained by the equation $\mathrm{nh}=(\mathrm{Nh} / \mathrm{N})^{*} \mathrm{n}$, where $\mathrm{nh}$ was the sample size of each outpatient clinic $\mathrm{h}$, Nh the population size (number of outpatients during a 3-month period at the specific hospital or dental health center) for stratum $h$, $\mathrm{N}$ the total population size (total number of outpatients during a 3-month period at all hospitals and dental health centers), and $n$ total sample size $(1,888)$. Full details of the sampling procedures have been published elsewhere (20). Patients were selected consecutively until the required number of patients from the different hospitals and dental health centers were enrolled.

Only 37 patients declined to participate or did not fulfill the inclusion criteria. Individuals were excluded from the study if they presented with severe malocclusion (e.g., prognathism), neurological disease, mental disability, or acute pain that could have interfered with the interview or clinical examination. Any subject with a history of rheumatic fever, heart murmur, endocarditis, or valvular heart disease, or with any artificial joint (usually hip or knee), did not undergo periodontal examination. The principal reasons for not participating were lack of time on the part of the patient and impatience to receive treatment.

Written consent was obtained from all patients. The National Ethical Clearance Committee of the Federal Ministry of Health in Khartoum, Sudan approved the study protocol (2009).

\section{Data collection}

\section{Interviews}

Data on sociodemographic, behavioral, and medical characteristics were collected by using a questionnaire administered in interviews by one of five calibrated dentists. Sociodemographic variables included age, sex, ethnic group, and socioeconomic status (occupation, total monthly income, education). Behavioral variables included frequency and reason for dental visits, number of teeth removed at final visit (if applicable), tobacco use and manner of consumption, and frequency and methods used for dental hygiene.

Because of developing interest in the relationship between oral and general health, we felt it necessary to ascertain some of the important medical characteristics 
of the population sample and thus included items on medication use, previous surgery, and details of diagnosed medical conditions. Participants were also asked "How often does your mouth feel dry?" and were given the following response options: "always", "frequently", "occasionally", and "never". For the purpose of analysis, those who responded "always" or "frequently" were classified as xerostomic. The interviews were completed within $15-20 \mathrm{~min}$ and were conducted in a confidential atmosphere before the clinical investigation.

The subjective impact of oral conditions was assessed in two ways. First, single, global items assessing perceived need for dental treatment were provided, using the response categories "no", "yes", and "don't know"; self-perceived oral and general health were assessed on an ordinal scale as "good", "fair", and "poor". Singleitem global self-ratings are short and have been used in larger population surveys (21). The disadvantage of such items is that they fail to meet the requirements of a multidimensional construct. In this study, they were used to determine convergent validity of the OHIP-14s-ar. Second, the shortened version of the Oral Health Impact Profile (OHIP-14), translated into Sudanese Arabic, was used to assess OHRQoL.

\section{Adaptation of the OHIP}

To create an Arabic version of the OHIP-14, translation guidelines using a forward-backward approach were followed based on previous studies $(22,23)$. For the forward translation, two teams of bilingual dentists first independently translated the English version of the OHIP-14. The dentists then collaborated to produce one common translation. For the back translation, two back-translations were independently produced by two bilingual translators working from the revised version of the questionnaire. Neither translator had access to the original English version of the questionnaire. A team consisting of the two Arabic linguistic experts and the forward translators then reviewed all the translations and reached a consensus on discrepancies. The team consolidated all versions of the questionnaire and developed the final version for field-testing. The final Arabic version was pilot-tested on a convenience sample of 20 participants. Each participant completed the questionnaire and was interviewed to reveal whether s/he understood the meaning of each questionnaire item and the chosen response, which was done to ensure that the colloquial Sudanese Arabic language version was appropriate. Responses were then reviewed by the committee. After reviewing the results, the final OHIP-14 Sudanese Arabic (s-ar) was revised accordingly.

The format of a typical question was as follows: "How often during the last 12 months have you had (impact item) because of problems with your teeth, mouth, or dentures?". Responses to each OHIP question were made on a Likert-type scale to indicate if the impact had occurred "never", "hardly ever", "occasionally", "fairly often", or "very often". For analysis, the responses were coded from 0 (for a response of "never") to 4 (for a response of "very often"). The total score was then calculated by summing responses of fairly often/very often $(17,24)$, and responses of occasionally/fairly often/ very often (25). Using this approach, total score ranged from 0 to 14 , and corresponding percentages or means were calculated. When computing OHIP scores, subjects with missing data for more than two items on the OHIP were excluded from the analysis. Higher scores indicate poorer OHRQoL.

\section{Clinical examination}

Five calibrated dentists, including the principal investigator, conducted the clinical examinations using dental units from the clinics, a dental mirror, and a WHO periodontal probe. The clinical examination included a full-mouth recording of the tooth status of 32 teeth using the decayed-missing-filled-teeth (DMFT) index. Decay was recorded if a carious cavity was visually present, and a community periodontal index (CPI) probe was used to confirm visual evidence of caries. In addition to dental status, periodontal health was assessed using the CPI; prosthetic status and need were established according to WHO criteria (26). The 12 upper and lower anterior teeth were examined for tooth wear $(27,28)$.

\section{Reliability}

Interexaminer reliability was assessed by calculating intraclass correlation coefficients (ICCs) for the clinical measures CPI, DMFT, and tooth wear, after an interval of 2-3 weeks. The ICCs for CPI, DMFT, and tooth wear were 0.67 (95\% CI, 0.56-0.83), 0.96 (95\% CI, 0.920.97 ), and 0.55 (95\% CI, 0.41-0.75), respectively, before the start of the survey, and 0.61 (95\% CI, 0.55-0.68), 0.85 (95\% CI, 0.81-0.87), and 0.59 (95\% CI, 0.49-0.62), respectively, during the survey. Thus, according to the criteria of Fleiss (29), the ICC was fair to good for CPI, excellent for DMFT, and fair to good for tooth wear.

\section{Data analysis}

Analyses were performed using the statistical software package STATA, Release 9 (Stata Statistical Software 2005; StataCorp LP, College Station, TX, USA). 


\section{Evaluation of OHIP}

Validity

Validity tests were carried out to ensure that the instrument was quantifying what it was intended to measure. Data from the first administration were used to assess the validity of the instrument. Because there was no "gold standard", construct validity was assessed. Two types of construct validity tests were performed: convergent and discriminant validity $(30,31)$.

Convergent validity describes how closely a measure is related to other measures of the same construct and was evaluated by identifying associations between perceived oral health status, general health status, perceived need for dental care, and OHIP scores. For convergent validity, we hypothesized that lower OHIP-14 scores would be associated with no perceived need for treatment and better self-reported oral and general health. We investigated the relationship between the 3 variables (self-rated oral health status, general health status, and perceived need for dental treatment) and OHIP-14 scores. The D'Agostino test of goodness-of-fit, which evaluates skewness and kurtosis, was used to test for normality and revealed a non-normal distribution of OHIP scores. Thus, we used the nonparametric Kruskal-Wallis 1-way analysis of variance, using ranks to assess associations between scores and other variables.

Discriminant validity assesses the extent to which a scale can distinguish between groups with known differences. We compared OHIP scores and the following clinical oral health variables: $\geq 21$ vs. $0-20$ teeth, 0 vs. $\geq 1$ missing teeth, $<.18$ vs. $\geq 18$ sound untreated natural teeth (SUNT), 0 vs. $\geq 1$ decayed teeth (DT), presence vs. absence of tooth wear, healthy periodontal tissues vs. presence of periodontal pockets $\geq 4 \mathrm{~mm}$, and presence vs. absence of a prosthesis. For discriminant validity, we hypothesized that higher OHIP-14 scores would be associated with decay, number of missing teeth, tooth wear, periodontal pockets $\geq 4 \mathrm{~mm}$, and prosthesis status. The associations between oral conditions and OHIP-14 scores were also examined using the Kruskal-Wallis test. Reliability

We examined 2 types of reliability in this study: internal consistency and test-retest reliability.

Internal consistency is a measure typically based on correlations between different items on the same test (or the same subscale of a larger test). We used Cronbach $\alpha$ to measure internal consistency (32). The Cronbach reliability coefficient was based on responses during the first administration and was used to determine whether removal of individual questions would increase the instrument's reliability.
Test-retest reliability was assessed by calculating ICCs, using scores from repeated administrations of the OHIP-14s-ar to 20 pilot patients. Two weeks elapsed between administrations of the test, and ICCs were calculated for OHIP subscales.

\section{Frequency distributions and bivariate and multivariate logistic regression analysis}

Data were analyzed by frequency distribution, and bivariate analysis was carried out using the Pearson $\chi^{2}$ test. The dependent variable of OHIP-14s-ar was dichotomized using the cut-off points never/hardly ever versus occasionally/fairly often/very often.

The independent categorical variables included sociodemographic characteristics (eg, sex, age group, ethnic group, occupation, monthly household income, and education level), behavioral measures (e.g., frequency of dental visits, tobacco use, and frequency and type of dental hygiene), medical variables (e.g., frequency of mouth dryness, surgical history, and presence of systemic illness).

Clinical variables were dichotomized into $\geq 21$ vs. 0 -20 teeth, 0 vs. $\geq 1$ missing teeth, $<18$ vs. $\geq 18$ SUNT, 0 vs. $\geq 1$ DT, presence vs. absence of tooth wear, healthy periodontal tissues vs. presence of periodontal pockets $\geq$ $4 \mathrm{~mm}$, and presence vs. absence of a prosthesis.

Finally, categorical variables of self-reported general health (good, fair, poor), oral health (good, fair, poor), and need for treatment (no, yes, don't know) were also included.

Only factors identified as significant by the Pearson $\chi^{2}$ test were entered into the multivariate logistic regression model, after which odds ratios (ORs) were calculated. ORs provide a more readily interpretable measure of the strength of association between the dependent and independent variables.

\section{Evaluation of OHIP}

\section{Results}

\section{Convergent validity}

Self-perceived oral and general health statuses, as well as need for treatment, were significantly associated $(P \leq$ 0.05 ) with total score on the OHIP-14s-ar regardless of whether scores were calculated using responses of fairly often/often or occasionally/fairly often/often (Table 1).

Discriminant validity

The oral health outcomes of tooth loss (i.e., $\geq 21$ vs. 0-20 teeth; 0 vs. $\geq 1$ missing teeth) were significantly associated with total score on the OHIP-14s-ar regardless of whether scores were calculated using responses of fairly often/often or occasionally/fairly often/often. 
Table 1 Convergent validity: OHIP-14s-ar scores by perceived need for dental treatment and general and oral health

\begin{tabular}{|c|c|c|c|c|c|c|c|}
\hline & $n(\%)$ & OHIP-14s-ar & & $\begin{array}{l}\text { Fairly often/ } \\
\text { very often }\end{array}$ & $\begin{array}{l}\text { Fairly often } \\
\text { or very often }\end{array}$ & $\begin{array}{l}\text { Occasionally, } \\
\text { fairly often, or } \\
\text { very often }\end{array}$ & $\begin{array}{l}\text { Occasionally, } \\
\text { fairly often, or } \\
\text { very often }\end{array}$ \\
\hline & & Mean (SD) & $P$ value & Mean (SD) & $P$ value & Mean (SD) & $P$ value \\
\hline \multicolumn{8}{|c|}{ Self-rated oral health } \\
\hline Good & $762(40.4 \%)$ & $4.5(4.8)$ & 0.0001 & $0.3(0.9)$ & 0.0001 & $1.3(2.0)$ & 0.0001 \\
\hline Fair & $698(37.0 \%)$ & $7.4(6.2)$ & & $0.6(1.4)$ & & $1.7(2.3)$ & \\
\hline Poor & $428(22.7 \%)$ & $11.8(9.0)$ & & $1.2(2.2)$ & & $3.9(3.0)$ & \\
\hline \multicolumn{8}{|c|}{ Self-rated general health } \\
\hline Good & $1310(69.4 \%)$ & $6.2(6.2)$ & 0.0001 & $0.5(1.3)$ & 0.0001 & $1.8(2.4)$ & 0.0001 \\
\hline Fair & $480(25.4 \%)$ & $8.6(7.5)$ & & $0.7(1.7)$ & & $2.1(2.7)$ & \\
\hline Poor & $96(5.1 \%)$ & $13.8(9.9)$ & & $1.3(2.3)$ & & $4.7(3.3)$ & \\
\hline \multicolumn{8}{|c|}{ Self-rated need for dental treatment } \\
\hline No & $151(8.0 \%)$ & $3.4(5.2)$ & 0.0001 & $0.2(0.7)$ & 0.0002 & $1.2(2.2)$ & 0.0001 \\
\hline Yes & $1688(89.4 \%)$ & $7.6(7.1)$ & & $0.6(1.5)$ & & $2.1(2.6)$ & \\
\hline Don't know & $49(2.6 \%)$ & $5.0(6.1)$ & & $0.3(1.3)$ & & $1.9(2.0)$ & \\
\hline
\end{tabular}

Kruskal-Wallis test

Table 2 Discriminant validity: OHIP-14s-ar scores by DMFT, CPI, missing teeth, and tooth wear

\begin{tabular}{|c|c|c|c|c|c|c|c|}
\hline & $n(\%)$ & OHIP-14s-ar & & $\begin{array}{l}\text { Fairly often/ } \\
\text { very often }\end{array}$ & $\begin{array}{l}\text { Fairly often } \\
\text { or very often }\end{array}$ & $\begin{array}{l}\text { Occasionally, } \\
\text { fairly often, or } \\
\text { very often }\end{array}$ & $\begin{array}{l}\text { Occasionally, } \\
\text { fairly often, or } \\
\text { very often }\end{array}$ \\
\hline & & Mean (SD) & $P$ value & Mean (SD) & $P$ value & Mean (SD) & $P$ value \\
\hline \multicolumn{8}{|c|}{ Decayed teeth (DT) } \\
\hline 0 & $232(12.3 \%)$ & $6.2(7.4)$ & 0.0001 & $0.6(1.6)$ & 0.6465 & $1.7(2.6)$ & 0.0033 \\
\hline$\geq 1$ & $1655(87.7 \%)$ & $7.4(7.0)$ & & $0.6(1.5)$ & & $2.1(2.6)$ & \\
\hline \multicolumn{8}{|l|}{ Missing teeth } \\
\hline 0 & $415(22 \%)$ & $6.3(6.4)$ & 0.0001 & $0.4(1.3)$ & 0.0053 & $1.7(2.4)$ & 0.0001 \\
\hline$\geq 1$ & $1473(78 \%)$ & $7.6(7.2)$ & & $0.6(1.6)$ & & $2.2(2.7)$ & \\
\hline \multicolumn{8}{|l|}{ Teeth present } \\
\hline$\geq 21$ & $1750(92.7 \%)$ & $7.0(6.8)$ & 0.0024 & $0.5(1.4)$ & 0.0001 & $2.0(2.5)$ & 0.0001 \\
\hline$<20$ & $138(7.3 \%)$ & $9.6(9.4)$ & & $1.1(2.2)$ & & $3.0(3.1)$ & \\
\hline \multicolumn{8}{|l|}{ Tooth wear } \\
\hline 0 & $1218(64.5 \%)$ & $6.9(6.7)$ & 0.2868 & $0.5(1.4)$ & 0.3523 & $1.9(2.4)$ & 0.4254 \\
\hline$>0$ & $670(35.5 \%)$ & $7.3(7.0)$ & & $0.6(1.5)$ & & $2.1(2.6)$ & \\
\hline \multicolumn{8}{|c|}{ Periodontal health } \\
\hline healthy gums & $781(41.4 \%)$ & $7.2(7.0)$ & 0.8185 & $0.6(1.5)$ & 0.9868 & $2.0(2.6)$ & 0.7428 \\
\hline pockets $\geq 4 \mathrm{~mm}$ & $174(9.2 \%)$ & $7.2(6.9)$ & & $0.6(1.5)$ & & $2.1(2.6)$ & \\
\hline \multicolumn{8}{|l|}{ Prosthesis } \\
\hline No & $1831(97 \%)$ & $7.2(7.0)$ & 0.2169 & $0.6(1.5)$ & 0.4073 & $2.0(2.6)$ & 0.0648 \\
\hline Yes & $57 \quad(3 \%)$ & $7.6(7.3)$ & & $0.6(1.6)$ & & $2.3(2.6)$ & \\
\hline
\end{tabular}

Kruskal-Wallis test, DMFT: decayed-missing-filled-teeth, CPI: community periodontal index

Tooth decay (0 vs. $\geq 1$ DT) was significantly associated with total score on the OHIP-14s-ar when scores were calculated using responses of occasionally/fairly often/ often (Table 2). OHIP-14s-ar score was not associated with tooth wear, periodontal pockets $\geq 4 \mathrm{~mm}$, or prosthesis status.

\section{Test-retest reliability}

The ICC for reliability among 20 participants who underwent assessment of the 7 OHIP-14s-ar subscales on two occasions ranged from 0.89 to 0.99 (average among raters) and from 0.8 to 0.97 (average for a typical rater) (Table 3). According to Shrout and Fleiss (33), an ICC > 0.75 indicates excellent reliability.

The Bland Altman (34), or difference, plot is a scatter diagram of the differences (OHIP-OHIP2) between the averages of two measurements and is used to examine test-retest reliability. Horizontal lines are drawn at the mean difference and at the limits of the agreement, which are defined as the mean difference \pm 1.96 times the SD of the differences. In the present study the arithmetic mean 
Table 3 Test-retest reliability, as measured by intraclass correlation coefficients (ICCs) for the OHIP-14s-ar and seven subscales

\begin{tabular}{|c|c|c|c|}
\hline \multicolumn{2}{|l|}{ Dimension (no. of items) } & $\mathrm{ICC}^{\mathrm{a}}$ & $95 \% \mathrm{CI}$ \\
\hline \multicolumn{4}{|l|}{ OHIP-14 } \\
\hline \multirow[t]{2}{*}{ Functional limitation (2) } & Single measures ${ }^{b}$ & 0.8 & $0.56-0.92$ \\
\hline & Average measures ${ }^{\mathrm{c}}$ & 0.89 & $0.72-0.96$ \\
\hline \multirow[t]{2}{*}{ Physical pain (2) } & Single measures ${ }^{\mathrm{b}}$ & 0.95 & $0.88-0.98$ \\
\hline & Average measures ${ }^{c}$ & 0.97 & $0.94-0.99$ \\
\hline \multirow[t]{2}{*}{ Psychological discomfort(2) } & Single measures ${ }^{\mathrm{b}}$ & 0.97 & $0.93-.99$ \\
\hline & Average measures ${ }^{c}$ & 0.99 & $0.96-0.99$ \\
\hline \multirow[t]{2}{*}{ Physical disability (2) } & Single measures ${ }^{\mathrm{b}}$ & 0.97 & $0.93-0.99$ \\
\hline & Average measures ${ }^{c}$ & 0.99 & $0.96-0.99$ \\
\hline \multirow[t]{2}{*}{ Psychological disability (2) } & Single measures ${ }^{\mathrm{b}}$ & 0.88 & $0.72-0.95$ \\
\hline & Average measures ${ }^{\mathrm{c}}$ & 0.94 & $0.84-0.97$ \\
\hline \multirow[t]{2}{*}{ Social disability (2) } & Single measures ${ }^{\mathrm{b}}$ & 0.8 & $0.56-0.92$ \\
\hline & Average measures ${ }^{\mathrm{c}}$ & 0.89 & $0.72-0.96$ \\
\hline \multirow[t]{2}{*}{ Handicap (2) } & Single measures ${ }^{\mathrm{b}}$ & 0.91 & $0.79-0.96$ \\
\hline & Average measures $^{\mathrm{c}}$ & 0.95 & $0.88-0.98$ \\
\hline \multicolumn{4}{|c|}{$\begin{array}{l}\text { a Degree of consistency among measurements. }{ }^{b} \text { Estimate of reliability } \\
\text { of single rating. }{ }^{c} \text { Estimate of reliability of average of } k \text { ratings. }\end{array}$} \\
\hline \multicolumn{2}{|l|}{ Dimension (no. of items) } & \multicolumn{2}{|c|}{ Cronbach $\alpha$} \\
\hline \multicolumn{2}{|l|}{ OHIP-14 } & \multicolumn{2}{|c|}{0.80} \\
\hline \multicolumn{2}{|l|}{ Functional limitation (2) } & \multicolumn{2}{|c|}{0.81} \\
\hline \multicolumn{2}{|l|}{ Physical pain (2) } & \multicolumn{2}{|c|}{0.78} \\
\hline \multicolumn{2}{|l|}{ Psychological discomfort (2) } & \multicolumn{2}{|c|}{0.75} \\
\hline \multicolumn{2}{|l|}{ Physical disability (2) } & \multicolumn{2}{|c|}{0.78} \\
\hline \multicolumn{2}{|l|}{ Psychological disability (2) } & \multicolumn{2}{|c|}{0.76} \\
\hline \multicolumn{2}{|l|}{ Social disability (2) } & \multicolumn{2}{|c|}{0.76} \\
\hline \multicolumn{2}{|l|}{ Handicap (2) } & \multicolumn{2}{|c|}{0.78} \\
\hline
\end{tabular}

was 0.01071 (95\% CI, -0.01 to 0.03$)$, the $\mathrm{SD}$ was 0.16 , the lower limit was -0.30 (95\% CI, -0.33 to 0.28 ), and the upper limit was 0.32 (95\% CI, 0.29 to 0.35 ). The limits of agreement represent the test-retest differences that can be expected for $95 \%$ of the subjects in the sample.

Internal consistency

Internal consistency (Cronbach $\alpha$ ) of the OHIP-14s-ar was assessed using the responses of the 1888 participants included in the survey. The Cronbach $\alpha$ for the OHIP$14 \mathrm{~s}$-ar was 0.81 , and $\alpha$ values for the seven subscales ranged from 0.75 to 0.78 (Table 4). According to Bland and Altman (35), a Cronbach $\alpha$ of $0.70-0.80$ is desirable.

\section{Distribution of responses to the OHIP scale}

Number of problems was ascertained by a simple count (SC) of impacts experienced during the previous year (Fig. 1). The proportion of respondents experiencing a problem was $50.3 \%$ (SC), using the cut-off point occa-

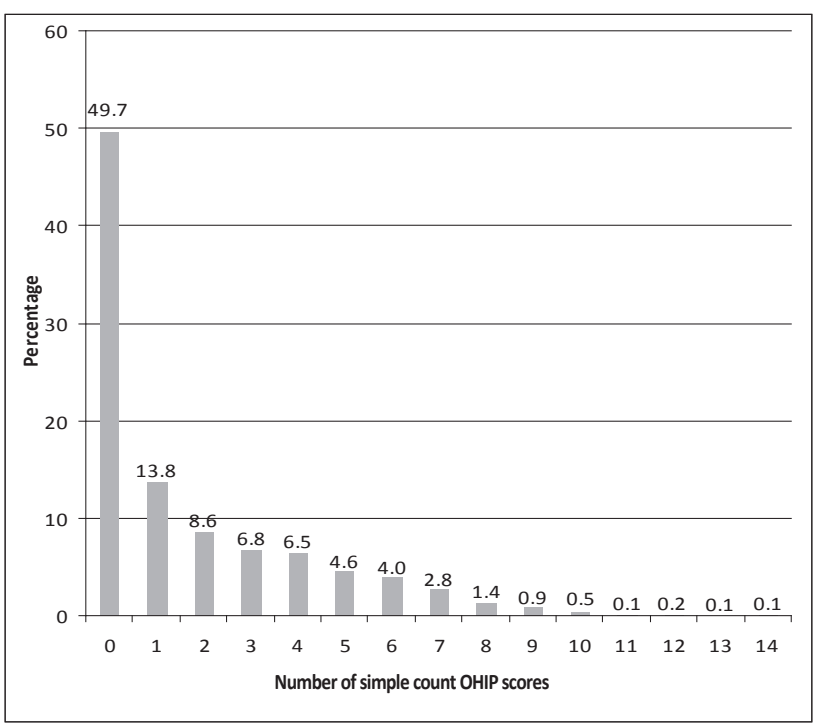

Fig. 1 Percentage of respondents by simple count of responses of occasionally/often/very often on OHIP-14s-ar items.

sionally/often/very often. The threshold "occasionally or more" was chosen to capture the responses of participants who had one impact episode. The types of impacts occasionally/fairly often/often experienced by adults are shown in Fig. 2; 47\% felt psychological discomfort (defined as feeling tense or self-conscious), and 7\% of those felt this way "very often". Some (37\%) felt physical pain from discomfort while eating or an aching mouth, and 5\% experienced this impact "very often". Psychological disability with difficulty in relaxing, or embarrassment due to their oral condition, was reported in $(26 \%)$ of the sample. Social disability (experiencing irritability or difficulty doing usual jobs) occurred in $22 \%$ of the sample, and $16 \%$ felt handicapped by their oral condition, as indicated by feeling that life was less satisfying or that they were unable to function due to their oral condition. Physical disability with interrupted meals or poor diet occurred in $15 \%$ of respondents. Functional limitations due to trouble pronouncing words or taste disturbances were encountered least, in $11 \%$ of the participants.

\section{Distribution of responses to global self-ratings}

Only $40 \%$ rated their oral health as good, as compared with $69 \%$ who rated their general health as good. Almost $89 \%$ reported needing dental treatment (Table 1).

\section{Characteristics associated with OHIP in Sudanese adults: Pearson $\chi^{2}$}

OHIP score was significantly associated with sex, frequency of dental visits, mouth rinsing, use of medication, mouth dryness, disease status, tooth loss, self-rated 


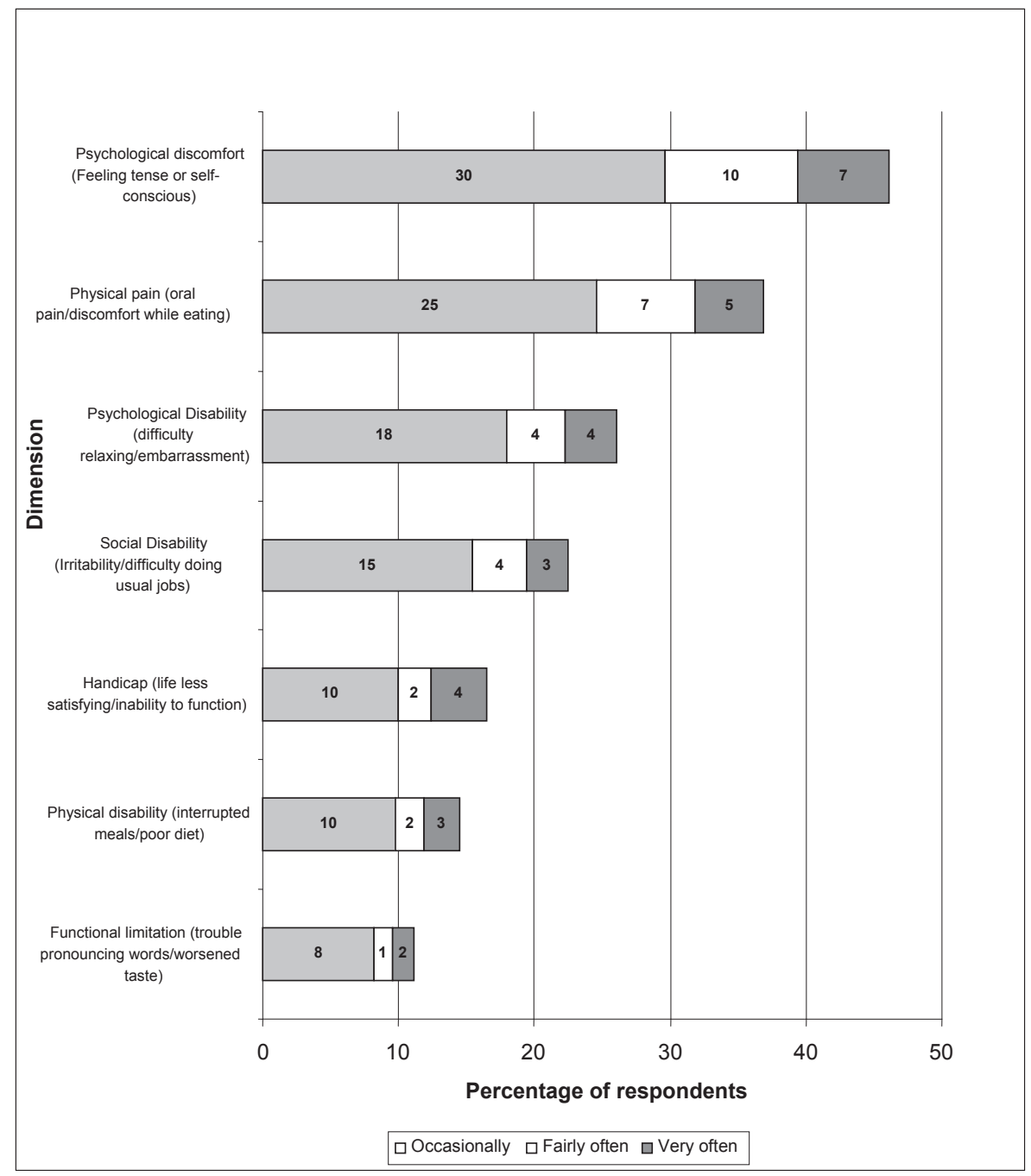

Fig. 2 Frequency of impacts reported by respondents.

oral and general health, and perceived need for dental treatment (Table 5).

\section{Characteristics associated with OHIP in Sudanese} adults: multivariate logistic regression analysis of the survey results

All independent factors found significant by Pearson $\chi^{2}$ analysis were entered in one step into a multivariate logistic regression model, along with the dichotomized OHIP-14s-ar variable as the dependent factor. Details of variables associated with statistically significant increases in the risk of oral disease are shown in Table 6. Females were significantly more likely $(\mathrm{OR}, 1.38)$ than males to present with reduced OHRQoL. Risk was also significantly higher among participants who reported dry mouth occasionally (OR, 1.77), frequently (OR, 2.67), or always (OR, 14.09). People with positive disease status $(\mathrm{OR}, 1.47)$ or tooth decay $(\mathrm{OR}, 1.44)$ rated their oral health fair $(\mathrm{OR}, 1.29)$ or poor $(\mathrm{OR}, 2.2)$. In addition, those who thought they required dental treatment (OR, 2.44) were more likely to report reduced QoL.

\section{Discussion}

Our findings suggest that people are affected in different ways by their oral condition. For some, the impact can be substantial. Prevalence and severity rates among dental patients using the OHIP-14s-ar are probably higher than those from a random population sample. Our sample consisted of people who already had a problem that led them to seek treatment.

The cross-cultural adaptation of the OHIP-14s-ar was done using published guidelines $(23,36,37)$. Even though the OHIP-49 had been previously translated into Arabic (22) and used in a Saudi population, cultural differences and difficulty in understanding some of the questions led us to develop a version more suitable for the Sudanese population. To our knowledge, this is the first time the OHIP-14 was used to investigate OHRQoL among Suda- 
Table 5 Characteristics associated with OHIP-14s-ar

\begin{tabular}{ll}
\hline Characteristic & $P$ value \\
\hline Age group & 0.300 \\
Sex & $0.000^{*}$ \\
Ethnic group & 0.951 \\
Occupation & 0.454 \\
Income & 0.375 \\
Education & 0.240 \\
Dental visits & $0.013^{*}$ \\
Reason for going to dentist & 0.091 \\
Tobacco use & 0.404 \\
Dental hygiene & \\
Toothbrushing & 0.804 \\
Mouth rinsing & $0.002^{*}$ \\
Cleaning between teeth & 0.075 \\
Use of medication & $0.006^{*}$ \\
Frequency of dry mouth & $0.000^{*}$ \\
Disease status & $0.000^{*}$ \\
$<18$ vs. $\geq 18$ sound teeth & $0.000^{*}$ \\
Tooth wear & 0.436 \\
Tooth loss (missing $\geq 1)$ & $0.014^{*}$ \\
$\geq 21$ vs. 0-20 teeth & $0.010^{*}$ \\
Prosthesis status & $0.017^{*}$ \\
Periodontal status & 0.812 \\
Decay & $0.002^{*}$ \\
Self-rated oral health & $0.000^{*}$ \\
Self-rated general health & $0.008^{*}$ \\
Self-perceived need for dental treatment & $0.000^{*}$ \\
\hline &
\end{tabular}

$* P<0.05$ significant, Pearson $\chi^{2}$ test

nese adults.

In consideration of the diversity of the sample, which included illiterate people, the questionnaire was administered as an interview. This principle has been applied in several studies $(10,24)$. Completion rates for the survey were excellent: less than $1 \%$ of the responses including missing items. Patient compliance was good, and the length of the OHIP-14s-ar seemed adequate as part of the lengthy questionnaire used for the whole survey. The main reason for using the short-form OHIP was to ease the burden on patients and clinicians. In addition, the findings of Slade (16) indicated that the OHIP-14 has good reliability, validity, and precision.

The Sudanese Arabic version of the OHIP-14s-ar preserved the overall concept of the original, English version. This study demonstrated that the OHIP is internally consistent (as shown by the Cronbach $\alpha$ ). The Cronbach $\alpha$ for internal consistency in this study was slightly lower than in studies by van der Meulen or John $(23,36)$, similar to values obtained by Slade and Spencer (10), and higher than those from Lopez (38).

The ICCs (33) calculated in our test-retest analyses were excellent, possibly because the interval between administrations of the questionnaire was only 2 weeks.
Table 6 Likelihood of a response of occasionally/fairly often/very often to an item on the OHIP-14s-ar, based on odds ratios (ORs) from logistic regression

\begin{tabular}{|c|c|c|}
\hline Characteristic & OR & $95 \% \mathrm{CI}$ \\
\hline \multicolumn{3}{|l|}{ Sex } \\
\hline Male & 1 & \\
\hline Female & $1.38^{* *}$ & $1.11-1.65$ \\
\hline \multicolumn{3}{|l|}{ Dry mouth } \\
\hline Never & 1 & \\
\hline Occasionally & $1.77 * *$ & $1.3-2.24$ \\
\hline Frequently & $2.67 * *$ & $0.91-4.43$ \\
\hline Always & $14.09^{*}$ & $-14.1-43.0$ \\
\hline \multicolumn{3}{|l|}{ Systemic illness } \\
\hline No & 1 & \\
\hline Yes & $1.47 *$ & $1.0-1.94$ \\
\hline \multicolumn{3}{|l|}{ Dental status (decay) } \\
\hline No decay & 1 & \\
\hline$\geq 1$ tooth with decay & $1.44 *$ & $1.01-1.87$ \\
\hline \multicolumn{3}{|l|}{ Self-rated oral health } \\
\hline Good & 1 & \\
\hline Fair & $1.29 *$ & $1.0-1.58$ \\
\hline Poor & $2.12 * *$ & $1.54-2.7$ \\
\hline \multicolumn{3}{|c|}{ Self-perceived need for dental treatment } \\
\hline No & 1 & \\
\hline Yes & $2.44 *$ & $1.52-3.36$ \\
\hline
\end{tabular}

Our values were slightly higher than those reported in other studies $(23,36)$. The adequate reliability on the OHIP-14s-ar was confirmed by the Bland-Altman plot (34), which showed that $95 \%$ of differences were within the limits of agreement.

The convergent and discriminant validity of the index is supported by the associations of the OHIP-14s-ar with a single-item rating of dental/general health, perceived need for treatment, and with three dental outcomes. The largest impact was found for the outcome having $<20$ teeth (mean OHIP score, 9.6; Table 2), which emphasizes the poorer OHRQoL in that group. Although we hypothesized that tooth wear, periodontal pockets $\geq 4 \mathrm{~mm}$, and prosthesis status would support the discriminant validity of the OHIPs-ar, these associations were not confirmed. These dental outcomes may have had little impact on OHRQoL because of the small number of people in this sample with moderate to severe tooth wear $(9.7 \%)$, periodontal pocketing $(9.2 \%)$, and prosthodontic appliances $(3 \%)$.

The prevalence of having one or more impacts at least occasionally was $50.3 \%$, and $14.7 \%$ reported impacts from five or more items. This is similar to findings reported for dentate adults in an adult dental health 
survey in the United Kingdom, where 51\% reported an impact occasionally to very often, and $11 \%$ reported five or more impacts (25).

Nearly $50 \%$ of people felt psychological discomfort, i.e., feeling tense or self-conscious. Physical pain due to an aching mouth or discomfort while eating was ranked second $(37 \%)$. This differs from the findings of a previous study (25), which found that pain was the most frequently encountered impact ( $40 \%$ of participants). Considering the nature of the outpatient clinics in Khartoum State, it is surprising that pain was not a more important issue.

We suspected that subjects would more frequently report functional limitations, discomfort, and pain than physical, psychological, and social disability (and that fewer still would experience a handicap). The results of the present model revealed that psychological discomfort and pain were the most frequently reported problems, followed by psychological and social disability, which were followed by experiencing a handicap. Physical disability and functional limitations were the least frequently reported problems. These findings differ from those of Nuttall et al. (25), whose results supported a model hierarchy in which experiencing a handicap was the least frequent impact. These differences might be due to slight differences in the conceptualization and interpretation of questions, dissimilarities in the characteristics of the populations, and/or differences in the perceived severity of oral health across cultures (39).

Even though the Pearson $\chi^{2}$ test showed significant associations between several characteristics and OHIPsar scores, only a few relationships remained significant when entered into the logistic regression model. In our logistic regression, no significant relationships were observed between age and OHIPs-ar scores. A study (13) of the impact of oral health on daily living showed that people lost more teeth as they grew older but that adults aged 70 years or older had better mean impact scores as compared with younger age groups. This might be because older adults have lower expectations for their oral health. An inverse relation between OHIP and age has been observed elsewhere $(40,41)$.

Poorer OHRQoL was observed in females, which is consistent with findings from a cohort study (14) that found that women generally reported poorer OHRQoL as compared with men for each OHIP-14 measure. Dry mouth was significantly associated with OHRQoL outcomes; this finding is similar to those from a study of older adults (age, 52-100 years) by Locker (42) and a study of younger adults (age, 32 years) by Thomson (43).

A significant relationship between presence of tooth decay and poorer OHRQoL has been previously reported
(25). As in our study, those who perceived their oral health as poor had worse OHRQoL (15). Significant associations between prevalence, extent, and severity scores on the OHIP-14 and three global ratings on the QoL measure were also observed by Locker et al. (44). Worsening of OHRQoL due to illness is understandable, as the oral complications of many systemic diseases can compromise QoL. Those who thought they needed dental treatment also had poorer OHRQoL, which indicates that patients are able to understand their own symptoms.

The most relevant finding of the present study is that dental caries was the medical condition with the greatest impact. In addition, tooth condition seemed to play a greater role than the actual absence of teeth.

In conclusion, we used the OHIP-14s-ar to measure OHRQoL. The OHIP-14s-ar had suitable convergent and discriminant validity and appropriate internal consistency (as indicated by Cronbach $\alpha$ ). This makes it suitable for cross-sectional and longitudinal studies. In addition to psychological discomfort, physical pain was a frequently experienced oral health impact, indicating that teeth need to be restored before decay becomes so advanced that it results in irreversible pulpal disease. Our results suggest that female sex, tooth decay, systemic illness, and dry mouth worsen OHRQoL. Strategies to improve general as well as oral health among Sudanese, especially for groups at high risk, could help reduce the effects of oral disorders on OHRQoL.

\section{Acknowledgments}

The Ministry of Health, Khartoum State, supported this research. Special thanks to Dr. Isam M. Ahmed for making this research possible.

\section{References}

1. Slevin ML, Plant H, Lynch D, Drinkwater J, Gregory WM (1988) Who should measure quality of life, the doctor or the patient? Br J Cancer 57, 109-112.

2. Allen PF (2003) Assessment of oral health related quality of life. Health Qual Life Outcomes 1, 40.

3. Cushing AM, Sheiham A, Maizels J (1986) Developing socio-dental indicators - the social impact of dental disease. Community Dent Health 3, 3-17.

4. Atchison KA, Dolan TA (1990) Development of the Geriatric Oral Health Assessment Index. J Dent Educ 54, 680-687.

5. Strauss RP, Hunt RJ (1993) Understanding the value of teeth to older adults: influences on the quality of life. J Am Dent Assoc 124, 105-110.

6. Locker D, Miller Y (1994) Subjectively reported oral health status in an adult population. Community Dent Oral Epidemiol 22, 425-430.

7. Leao A, Sheiham A (1996) The development of a socio-dental 
measure of dental impacts on daily living. Community Dent Health 13, 22-26.

8. Adulyanon S, Vourapukjaru J, Sheiham A (1996) Oral impacts affecting daily performance in a low dental disease Thai population. Community Dent Oral Epidemiol 24, 385-389.

9. Grath CM, Bedi R, Gilthorpe MS (2000) Oral health related quality of life - views of the public in the United Kingdom. Community Dent Health 17, 3-7.

10. Slade GD, Spencer AJ (1994) Development and evaluation of the Oral Health Impact Profile. Community Dent Health $11,3-11$.

11. Locker D (1988) Measuring oral health: a conceptual framework. Community Dent Health 5, 3-18.

12. World Health Organization (1980) International classification of impairments, disabilities and handicaps: a manual of classification relating to the consequences of disease. WHO, Geneva, 1-205.

13. Steele JG, Sanders AE, Slade GD, Allen PF, Lahti S, Nuttall $\mathrm{N}$ et al. (2004) How do age and tooth loss affect oral health impacts and quality of life? A study comparing two national samples. Community Dent Oral Epidemiol 32, 107-114.

14. Mason J, Pearce MS, Walls AW, Parker L, Steele JG (2006) How do factors at different stages of the lifecourse contribute to oral-health-related quality of life in middle age for men and women? J Dent Res 85, 257-261.

15. Sanders AE, Slade GD, Lim S, Reisine ST (2009) Impact of oral disease on quality of life in the US and Australian populations. Community Dent Oral Epidemiol 37, 171-181.

16. Slade GD (1997) Derivation and validation of a short-form oral health impact profile. Community Dent Oral Epidemiol 25, 284-290.

17. Slade GD, Nuttall N, Sanders AE, Steele JG, Allen PF, Lahti S (2005) Impacts of oral disorders in the United Kingdom and Australia. Br Dent J 198, 489-493.

18. Elsharif MM (2006) Prosthodontic treatment need in military hospital. Master of Science Thesis, University of Khartoum, Khartoum, 1-112.

19. Ali MY (2009) Prosthodontic treatment need among adult dental patients attending the federal state hospitals and health centers in Khartoum State. Master of Science Thesis, University of Khartoum, Khartoum, 1-75.

20. Khalifa N, Allen PF, Abu-bakr NH, Abdel-Rahman ME, Abdelghafar KO (2012) A survey of oral health in a Sudanese population. BMC Oral Health 12, 5.

21. Gift HC, Atchison KA, Drury TF (1998) Perceptions of the natural dentition in the context of multiple variables. J Dent Res 77, 1529-1538.

22. Al-Jundi MA, Szentpétery A, John MT (2007) An Arabic version of the Oral Health Impact Profile: translation and psychometric properties. Int Dent J 57, 84-92.

23. van der Meulen MJ, John MT, Naeije M, Lobbezoo F (2008) The Dutch version of the Oral Health Impact Profile (OHIP$\mathrm{NL}$ ): translation, reliability and construct validity. BMC Oral Health 8, 11 .

24. Slade GD, Spencer AJ, Locker D, Hunt RJ, Strauss RP, Beck
JD (1996) Variations in the social impact of oral conditions among older adults in South Australia, Ontario, and North Carolina. J Dent Res 75, 1439-1450.

25. Nuttall NM, Steele JG, Pine CM, White D, Pitts NB (2001) The impact of oral health on people in the UK in 1998. Br Dent J 190, 121-126.

26. World Health Organization (1997) Oral health surveys: basic methods. 4th ed, WHO, Geneva, 36-47.

27. Nunn J, Morris J, Pine C, Pitts NB, Bradnock G, Steele J (2000) The condition of teeth in the UK in 1998 and implications for the future. Br Dent J 189, 639-644.

28. Office for National Statistics (2000) Adult dental health survey oral health in the United Kingdom in 1998. Stationery Office Books, London, 74-76.

29. Fleiss J (1986) The design and analysis of clinical experiments. Wiley, New York, 17-28.

30. Saub R, Locker D, Allison P (2005) Derivation and validation of the short version of the Malaysian Oral Health Impact Profile. Community Dent Oral Epidemiol 33, 378-383.

31. Atieh MA (2008) Arabic version of the Geriatric Oral Health Assessment Index. Gerodontology 25, 34-41.

32. Cronbach LJ (1951) Coefficient alpha and the internal reliability of tests. Psychometrika 16, 297-334.

33. Shrout PE, Fleiss JL (1979) Intraclass correlations: uses in assessing rater reliability. Psychol Bull 86, 420-428.

34. Bland JM, Altman DG (1986) Statistical methods for assessing agreement between two methods of clinical measurement. Lancet 1, 307-310.

35. Bland JM, Altman DG (1997) Cronbach's alpha. BMJ 314, 572.

36. John MT, Patrick DL, Slade GD (2002) The German version of the Oral Health Impact Profile - translation and psychometric properties. Eur J Oral Sci 110, 425-433.

37. Montero-Martín J, Bravo-Pérez M, Albaladejo-Martínez A, Hernández-Martín LA, Rosel-Gallardo EM (2009) Validation the Oral Health Impact Profile (OHIP-14sp) for adults in Spain. Med Oral Patol Oral Cir Bucal 14, E44-50.

38. Lopez R, Baelum V (2006) Spanish version of the Oral Health Impact Profile (OHIP-Sp). BMC Oral Health 6, 11.

39. Allison P, Locker D, Jokovic A, Slade G (1999) A crosscultural study of oral health values. J Dent Res 78, 643-649.

40. Robinson PG, Gibson B, Khan FA, Birnbaum W (2003) Validity of two oral health-related quality of life measures. Community Dent Oral Epidemiol 31, 90-99.

41. Slade GD, Sanders AE (2011) The paradox of better subjective oral health in older age. J Dent Res 90, 1279-1285.

42. Locker D (2003) Dental status, xerostomia and the oral health-related quality of life of an elderly institutionalized population. Spec Care Dentist 23, 86-93.

43. Thomson WM, Lawrence HP, Broadbent JM, Poulton R (2006) The impact of xerostomia on oral-health-related quality of life among younger adults. Health Qual Life Outcomes 4, 86.

44. Locker D, Quiñonez C (2011) To what extent do oral disorders compromise the quality of life? Community Dent Oral Epidemiol 39, 3-11. 